FORMATION Formation emploi

Revue française de sciences sociales

104 | octobre-décembre 2008

Pêle-mêle

\title{
Bihr A. et Pfefferkorn R. (2008), Le système des inégalités
}

La Découverte, coll. « Repères »

Henri Eckert

\section{(2) OpenEdition}

\section{Journals}

Édition électronique

URL : http://journals.openedition.org/formationemploi/1766

DOI : 10.4000/formationemploi.1766

ISSN : 2107-0946

Éditeur

La Documentation française

Édition imprimée

Date de publication : 1 octobre 2008

Pagination : 83-87

ISSN : 0759-6340

\section{Référence électronique}

Henri Eckert, «Bihr A. et Pfefferkorn R. (2008), Le système des inégalités », Formation emploi [En ligne],

104 | octobre-décembre 2008, mis en ligne le 23 février 2009, consulté le 30 octobre 2020. URL

http://journals.openedition.org/formationemploi/1766 ; DOI : https://doi.org/10.4000/

formationemploi.1766

(c) Tous droits réservés 


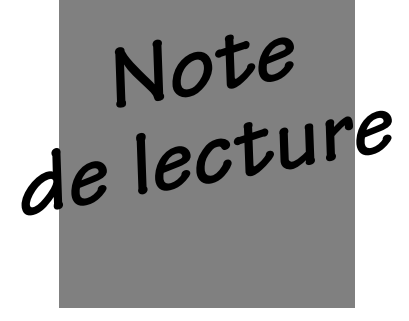

\section{Présentation de l'ouvrage "Le système des inégalités »}

Par Henri Eckert ${ }^{*}$

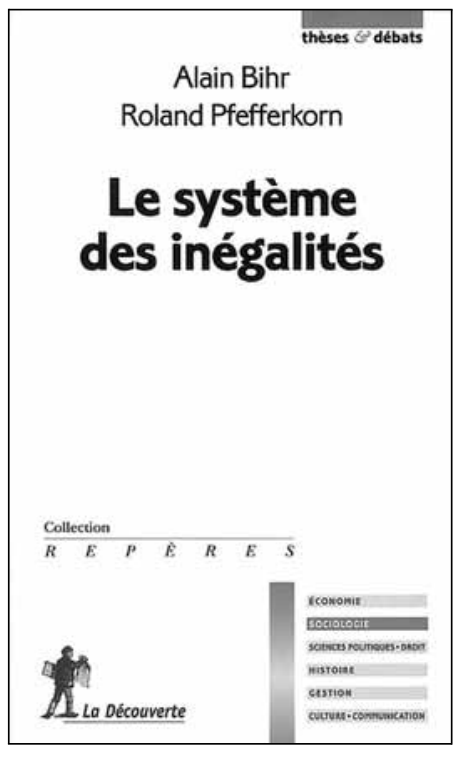

Les inégalités sociales feraient-elles système? Concentreraient-elles leurs effets délétères en certaines zones de l'espace social, affectant de préférence certains groupes sociaux, infligeant des manques divers aux uns pendant que les autres bénéficieraient de tous les atouts? La question se pose avec d'autant plus d'acuité que, malgré l'intuition diffuse d'un cumul par certains groupes sociaux des effets négatifs des inégalités sociales, la focalisation des études sur tel ou tel type d'inégalités - réussite scolaire contrastée des élèves selon leur origine sociale ou disparités de revenus entre hommes et femmes à compétence égale, par exemple - conduit trop souvent à négliger de mettre les inégalités observées dans un domaine particulier en relation avec celles repérées dans des domaines adjacents. D'où l'exigence, formulée d'entrée de jeu par les auteurs de cet ouvrage, d'une "approche systémique des inégalités sociales» (p. 6), susceptible, précisément, de mettre en évidence les relations que ces inégalités entretiennent entre elles. Arrêtonsnous plus longuement sur le projet des auteurs, d'autant qu'ils y consacrent le premier chapitre de l'ouvrage.

\section{LES ENJEUX \\ D'UNE APPROCHE SYSTÉMIQUE}

Multiformes, les inégalités concernent trois grands registres de la vie sociale : celui de l'avoir, bien sûr, mais aussi les registres du savoir et du pouvoir. Si les inégalités dans l'ordre de l'avoir ont conduit à une approche quantitative, le «simple relevé des données chiffrées concernant différentes inégalités sociales ne saisit pas et risque même d'occulter leur caractère systémique»(p. 10). Les inégalités ne sont d'ailleurs pas toujours mesurables et, quand elle s'avère possible, leur mesure ne permet pas forcément d'en rendre compte intégralement et peut, ainsi, en dissimuler les autres aspects. Surtout, la mesure survenant en aval du fait lui-même, s'en tient en quelque sorte à sa surface, quand il s'avère nécessaire d'en rechercher ses causes profondes. Or les

* Henri Eckert est sociologue, chargé d'études au Céreq. II travaille sur l'insertion sociale et professionnelle des jeunes. II a publié récemment: "Avoir vingt ans à l'usine», La Dispute, Paris, 2006. 
inégalités sociales, en tant que telles, sont "l'œuvre de la société dans laquelle on [les] constate» (p. 11) et il convient donc d'en rechercher les causes ultimes "dans le tréfonds des relations fondamentales que les hommes entretiennent entre eux» (p. 11). À ce point, il s'avère nécessaire de poser la question des rapports entre inégalités et injustices : si les inégalités font souvent naître un sentiment d'injustice, de révolte ou de compassion pour celui qui en est victime, certains discours ont visé à les légitimer. Les auteurs évoquent ainsi trois grands discours de légitimation des inégalités sociales : s'ils passent rapidement sur les discours qui naturalisent les inégalités, ils s'attardent plus longuement sur les deux autres types de discours, qui articulent chacun à sa manière la revendication d'une égalité formelle entre les citoyens avec le constat de l'inéluctabilité des inégalités de fait entre les agents économiques.

Pour certains, l'activité économique produit inévitablement des inégalités entre des agents égaux en droit et ces inégalités, parfois jugées accessoires, sont déclarées nécessaires à l'efficacité économique et à la liberté politique et s'en trouvent ainsi justifiées. Si les auteurs s'attachent à démonter les arguments avancés par les tenants de cette position - et consacrent quelques pages particulièrement pertinentes au thème de "l'égalité des chances ", qu'ils qualifient fort à propos de "curieux oxymore" (p. 18) -, ils mettent encore davantage de soin à rejeter la thèse de Rawls, selon laquelle l'inégalité constituerait un mal dont naîtrait un bien. Pour ce dernier, " une inégalité économique ou sociale serait justifiée dès lors qu'elle profiterait à ceux-là même qui la subissent; si bien que le sort des moins bien lotis pourrait être meilleur dans une société inégalitaire que dans une société égalitaire» (pp. 21/22). Or, nous expliquent les auteurs, "l'argumentation rawlsienne est spécieuse en ce qu'elle compare une situation réelle à une situation hypothétique, que l'on peut toujours choisir de telle manière ou représenter de telle façon que c'est la première qui apparaîtra préférable»(p.22). Mais ils la rejettent en outre parce que, «si l'argumentation rawlsienne peut à la rigueur avoir un sens pour des inégalités dans l'ordre de l'avoir, elle perd toute portée en ce qui concerne les inégalités dans l'ordre du pouvoir ou dans l'ordre du savoir » (p. 22). Enfin, si « les inéga- lités économiques et sociales tendent à annuler les effets correcteurs des dispositifs visant à garantir 1'"égalité des chances", elles menacent tout aussi bien l'égalité d'accès aux libertés fondamentales qui est à la base de l'édifice rawlsien » (p. 23).

Une fois repoussés les discours de légitimation des inégalités sociales, il est possible d'en revenir à l'argumentaire sur la systématicité des inégalités sociales. S'il importe d'étayer une approche critique des inégalités sociales sur "une mesure chiffrée de ces dernières » (p. 23), l'enjeu n'est pas tant d'établir l'existence des inégalités elles-mêmes - largement avérée par les nombreuses études portant sur telle ou telle forme d'inégalité - que de montrer comment "ces inégalités forment un système » (p. 28) Cet objectif implique ainsi "d'établir successivement que les inégalités interfèrent entre elles, en se déterminant et même en se générant réciproquement; qu'elles tendent par conséquent à former un processus cumulatif, au terme duquel les privilèges s'accumulent à l'un des pôles de l'échelle sociale tandis que, à l'autre pôle, s'accumulent les handicaps, cette accumulation et cette polarisation constituant des propriétés originales du système des inégalités; que se dessine ainsi une structure (un ensemble cohérent) relativement permanente dotée d'une forte capacité de se reproduire de génération en génération » (p. 29). Voilà le programme déroulé : les chapitres II, III et IV vont donc aborder successivement les interactions entre inégalités, leur tendance au cumul et, enfin, leur mode de reproduction. Tout au long de ce parcours, les auteurs prennent soin de s'appuyer sur les données statistiques disponibles : il est vrai qu'ils avaient entrepris, par le passé, une recension minutieuse et précise des inégalités observées dans les principaux domaines de la vie sociale française, parue sous un titre explicite : «Déchiffrer les inégalités » et mise à jour en 1999.

\section{LES INÉGALITES EN LEUR SYSTÈME}

Les interactions entre inégalités pourraient être résumées par cette formule en miroir : un même effet, de multiples causes versus une même cause, de multiples effets. C'est, du moins, ce que tendent à 
montrer les deux exemples présentés par les auteurs au début de leur deuxième chapitre. Ainsi, les inégalités face à la santé sont, plus ou moins directement, déterminées par d'autres inégalités : les conditions dans lesquelles les individus travaillent - ou plus exactement les inégalités des conditions de travail constituent ainsi l'une des causes principales des inégalités de santé. Mais les différences de modes de vie ou les inégalités d'accès aux soins n'en jouent pas moins leur rôle. À l'inverse, les inégalités face au logement induisent ou influent sur d'autres types d'inégalités pour, bien souvent, les aggraver, qu'il s'agisse de la réussite scolaire des enfants, variable selon qu'ils disposent ou non d'une chambre individuelle par exemple, des possibilités d'accéder à certains services, inégalement répartis sur le territoire, ou des risques pour la santé des individus. Ces interactions entre divers types d'inégalités peuvent être représentées schématiquement sur un tableau synoptique (p. 46) qui les situe à la fois comme variables explicatives et variables expliquées, l'ensemble des interactions étant matérialisé par des signes conventionnels, en l'occurrence des + ou des - . Ce tableau permet non seulement de donner à voir comment les inégalités tendent généralement « $\grave{a}$ se renforcer» (p. 47) mais aussi d'en évaluer la hiérarchie, dans la mesure où il laisse entrevoir quelles inégalités sont "le plus fréquemment en situation de facteur déterminant par rapport aux autres » (p. 52).

Si les inégalités de position au sein des rapports de production figurent le plus souvent « en position de facteur déterminant de premier rang 》 (id.), celles-ci semblent tenir avant tout aux inégalités de patrimoine et aux inégalités face à l'école, deux types majeurs d'inégalités qui pourraient ainsi jouer un rôle décisif dans la reproduction des inégalités dans leur ensemble. Mais cette configuration détermine aussi "l'accumulation des handicaps d'un côté et des privilèges de l'autre » (p. 55) et contribue ainsi à «la polarisation sociale» (id.). Un tableau synoptique des « indices d'inégalité entre catégories socioprofessionnelles » (pp. 56-57) donne à voir cette polarisation: les catégories du salariat d'exécution apparaissent très largement défavorisées au regard de critères tels que le taux d'emploi stable, les divers types de revenus, le patrimoine, le logement ou encore la réussite scolaire des enfants. La pauvreté ne se limite pas, en effet, "au défaut d'avoir » (p. 60), elle se révèle aussi dans «le défaut de pouvoir » (id.) et "le défaut de savoir» (p. 61), si bien qu'elle peut être définie, "précisément par l'accumulation de handicaps (de défauts ou de déficits) résultant d'inégalités tendant à se renforcer réciproquement " (id.). À l'opposé, la richesse se présente de même "comme une réalité embrassant l'ensemble des aspects de l'existence individuelle et collective» (p. 65). Mais, au-delà des effets de polarisation produits par le cumul des effets des inégalités sur certaines catégories sociales, ces inégalités tendent à croître depuis le milieu des années 80. C'est du moins ce que suggère l'évolution du « baromètre des inégalités et la pauvreté ou BIP40» (p. 73), un indicateur synthétique des inégalités sociales dans la société française.

Si l'accroissement des inégalités constitue une préoccupation actuelle, leur reproduction constitue un défi permanent pour des sociétés démocratiques, censées régler la distribution des individus dans l'espace social selon un critère de mérite. Or les études de mobilité sociale - intergénérationnelle en l'occurrence - induisent le doute sur ce fonctionnement méritocratique. Malgré l'augmentation de la mobilité sociale qui résulte des transformations de la structure des emplois d'une génération à l'autre - de nature à conforter une apparente fluidité sociale - «l'hérédité sociale semble peser à nouveau davantage» (p. 92). De fait, "par le biais du capital culturel et scolaire qu'elle transmet, chaque catégorie sociale détermine puissamment la destinée des individus qui en sont issus : plus l'origine sociale d'un individu est élevée, plus il aura de chances d'atteindre un niveau d'études élevé et, compte tenu de la liaison forte entre formation et emploi, plus il pourra atteindre lui-même une position sociale élevée. Et l'inverse se vérifie tout autant... » (p.97). Le thème a certes été rebattu, et renvoyer à des thèses anciennes (Bourdieu et Passeron, 1970; Baudelot et Establet, 1972) paraît, aux yeux de certains du moins, lui dénier toute pertinence actuelle. Or, comme par le passé, l'école continue de remplir "une fonction d'instance de reproduction d'un ordre social donné» (p. 98). Le rendement social des études - certains travaux du Céreq le confirment - continue de dépendre large- 
ment de l'origine sociale des individus, soit que les enfants issus de milieux sociaux favorisés réussissent mieux dans leurs études, soit qu'ils sont en mesure de tirer un meilleur parti du diplôme qu'ils détiennent et, généralement, pour ces deux raisons réunies.

\section{S'EN TENIR AUX INÉGALITÉS?}

Si la démonstration menée par les deux auteurs de l'ouvrage est d'autant plus convaincante qu'elle est abondamment étayée, reste pourtant cette question : faut-il s'en tenir aux inégalités, même après avoir pris le soin d'expliciter comment elles font système ? Pour paraphraser les auteurs: la seule approche systémique des inégalités pourrait ne pas saisir et risque même d'occulter le système de domination. Soyons clairs: la question n'invalide en rien la démonstration. Elle constitue bien davantage une invite à s'interroger plus avant sur le mécanisme de reproduction des inégalités évoqué par les auteurs. Pour en comprendre le fonctionnement, ne faut-il pas introduire aussi dans le raisonnement les aspects de domination qui vont de pair avec les inégalités? Certes, le mot « domination » et ses dérivés sont loin d'être absents de l'ouvrage mais il nous semble que les auteurs hésitent à en souligner les ressorts et l'impact.

Or la persistance de rapports inégalitaires, voire leur aggravation, ne nous paraît pas tenir seulement au mécanisme de leur reproduction. Au-delà du mécanisme lui-même, c'est toute la question de la légitimation de l'ordre social qui se trouve posée et, à travers elle de la possibilité même de la domination. Dans des «sociétés conflictuelles» (p. 105), le «mécanisme» de reproduction des inégalités ne suffit pas à maintenir les déséquilibres qui assurent à certaines catégories des jouissances au prix des privations subies par d'autres. La puissance qu'il requiert ne se constitue comme telle qu'au prix d'un travail de légitimation d'elle-même et peut alors seulement assurer la domination d'une catégorie sociale et la reproduction des inégalités dont elle se nourrit. Au moment de discuter les thèses rawlsiennes notamment, les auteurs entrent dans un débat de légitimité ; sans doute leur livre aurait-il encore gagné à aller plus loin dans ce sens. Mais peut-être le format du livre ne le permettait-il pas?

Ainsi faut-il retenir de ce livre précieux, outre son apport à la question de la systématicité des inégalités, l'appel à revenir sur l'existence et la perpétuation des inégalités dans la société française d'aujourd'hui, trop souvent niées ou acceptées comme inéluctables. Au-delà des discours sur la "moyennisation» des sociétés postindustrielles et malgré le triomphe proclamé de l'« individu », il s'agit d'en revenir à ce qui fait lit aux diverses formes de violence au sein de la société - l'ouvrage de Stéphane Beaud et Michel Pialoux reliant violences urbaines et violence sociale (2003) en constitue un superbe exemple -, dont la raison profonde pourrait bien tenir aux formes de domination et aux inégalités qu'elles légitiment et entretiennent. Qu'il s'agisse de formes de domination ou d'inégalités liées aux positions dans le système de production ou aux rapports de sexe (Pfefferkorn, 2007).

\section{Référence de l'ouvrage}

Bihr A. et Pfefferkorn R. (2008), Le système des

inégalités, La Découverte, coll. « Repères », 


\section{Bibliographie}

Baudelot C. et Establet R. (1972), L'école capitaliste en France, Maspero, Paris.

Beaud S. et Pialoux M. (2003), Violences urbaines, violence sociale - genèse des nouvelles classes dangereuses, Fayard, Paris.

Bihr A. et Pfefferkorn R. (1999 pour la 2e édition), Déchiffrer les inégalités, Syros, Paris.
Bourdieu P. et Passeron J.-C. (1970), La reproduction, Les Éditions de Minuit, Paris.

Pfefferkorn R. (2007), Inégalités et rapports sociaux. Rapports de classes, rapports de sexes, La Dispute, Paris. 